\section{Injected Dose in Pediatric PET}

TO THE EDITOR: In their recent paper, Accorsi et al. (1) provide a detailed quantitative analysis of the efficacy of pediatric patient injected dose in ${ }^{18} \mathrm{~F}$-FDG PET. For their analysis, they have defined a quantitative metric of data quality, the "noiseequivalent count density" (NECD), as a surrogate for clinical image quality. NECD is the total noise-equivalent counts (NEC) in the measured data divided by an estimate of the volume of the patient within the field of view of the scanner. I believe that in general the use of such a data quality measure as a proxy for imaging performance is a valuable approach for guiding scanning protocols. As the authors mention, NEC has been widely used for this purpose in other contexts. I would like to point out, however, that NECD is a metric qualitatively different from NEC and that its use leads to dose recommendations significantly different from what would have been derived from using NEC as the metric.

The motivation for characterizing data quality in terms of NECD rather than NEC is not spelled out in the paper. My tentative assumption is that the goal is to approximately equalize the signal-to-noise ratio (SNR) per unit image volume. Although there are many confounding issues, intuitively one might suppose that if the number of (noise-equivalent) counts per unit volume were the same for 2 patients, then the SNR (mean divided by the $\mathrm{SD}$ of the noise) for volumes of interest of the same size in the 2 images would likely be similar on average. Thus, pediatric and adult scans performed with similar NECDs might be expected to have similar SNRs in anatomically matched regions of equal volume-which may seem to be a valid criterion for equivalent image quality.

I would question such an assumption, however. It seems to me that tissue volumes of clinical interest are in general more likely to scale with the size (volume) of the patient. Many disease processes imaged in PET tend to be primarily associated with a specific organ (such as the heart), and children have smaller organs than adults. And, although I have no hard evidence supporting this hypothesis, it also seems plausible that the physiologic impact of a cancerous neoplasm would scale at least to some extent with the size of the body and, thus, that the detection of smaller tumors in smaller bodies would be required for equivalent efficacy.

Roughly speaking, an equalized NEC-based regimen would tend to equalize SNR per organ rather than per volume. Therefore, to the extent that clinically meaningful volumes of interest also scale with organ or body size, I believe that NEC may be a better predictor of image quality than is NECD. I therefore find the authors' statement (page 296) that “... the current [weight-based] injection regimen produces better image quality in lighter patients..." doubtful. It seems more probable to me that the recommended constant-NECD regimen would produce poorer image quality in lighter patients.

Certainly, minimizing unnecessary injected dose in pediatric PET is an extremely important goal, and the authors are to be commended for their careful analytic approach to it. The equalized NECD regimen they propose would in fact result in much smaller doses (approximately proportional to the patient's weight) than

COPYRIGHT @ 2010 by the Society of Nuclear Medicine, Inc. would a similar strategy based on NEC. My concern is that this regimen carries the risk that injected dose could be reduced to the point that the clinical value of the PET scan does not, in the end, justify the deleterious effects of the radiation absorbed by the patient. I believe it would be most prudent to maintain NEC as high as reasonably possible until there is solid evidence that diagnostic image quality is adequately estimated by NECD.

A second fundamental assumption underlying this paper is that the NECD observed for a 3-min scan of a 70-kg adult on the Gemini 16 PET/CT scanner (Philips Health Care) with a 363-MBq injected dose is clinically good enough and that any excess NECD can be traded off for lower dose or shorter scanning time. Yet I have never seen a convincing clinical study quantifying how many noise-equivalent counts are enough in PET. I believe that any PET image can be improved by additional valid data and, thus, that it is in the patient's best interest, when scan time is not limited by radiopharmaceutical redistribution or decay, to scan every patient for as long as is practical, regardless of dose. Further, for ${ }^{18} \mathrm{~F}-\mathrm{FDG}$ imaging I see no reason to limit the injected dose to less than that estimated to achieve about $90 \%$ of peak noise equivalent count rate unless the health risk of the absorbed radiation outweighs the likely clinical benefit of the scan. Unfortunately, as we know, this criterion is exceedingly difficult to quantify. Yet I believe that the definition of a more efficacious dose regimen can come only from a better understanding of this trade-off.

\section{REFERENCE}

1. Accorsi R, Karp JS, Surti S. Improved dose regimen in pediatric PET. J Nucl Med. 2010;51:293-300.

\section{Charles C. Watson}

Siemens Medical Solutions USA, Inc.

810 Innovation Dr.

Knoxville, TN 37932-2562

E-mail: charles.c.watson@siemens.com

DOI: 10.2967/jnumed.110.078493

REPLY: We would like to thank Dr. Watson for his interest in and comments about our paper (1).

The main concern raised is the rationale behind the choice of noise-equivalent count density (NECD) over noise-equivalent count (NEC). The choice was indeed guided by the logic inherent in equalizing signal-to-noise ratio (SNR) per unit image volume. However, the use of NEC to represent image quality in a clinical PET study is premised on its being a global measure of the data quality obtained in the study. Consequently, we think that NECD represents a reasonable global metric to equalize the general image quality between different-sized patients (children and adults) if the image quality is high enough in large patients. Any effort to measure the effects of disease in specific organs or cancerous neoplasms will be sensitive to local effects in the images (such as spatial resolution, image reconstruction, and data corrections), and so the choice of either NEC or NECD as a surrogate for any form of image quality index should be used with 
caution, especially given that little supporting evidence is available for either option.

A second concern relates to the use of the Gemini 16 PET/CT scanner (Philips Health Care), as well as the manufacturer's recommended dose regimen that was followed. Our work was performed on only 1 scanner, admittedly no longer state-of-the-art, but the technique (based on Dr. Watson's original work) should be independent of the performance of that scanner. Based on input from our clinical colleagues, images obtained from the Gemini 16 are clinically useful in large patients and, commonly, better in light patients for the same scanning time and weight-based dose regimen. As a result, we believe that using the peak or close to the peak (e.g., 90\%) NECD value in adults as a guideline to derive specific regimens for pediatric studies is justified.

\section{REFERENCE}

1. Accorsi R, Karp JS, Surti S. Improved dose regimen in pediatric PET. J Nucl Med. 2010;51:293-300.

\section{Roberto Accorsi* \\ Joel S. Karp \\ Suleman Surti}

*via Milite Ignoto 380

Ispra 21207, Italy

E-mail: raccorsi71@gmail.com

DOI: 10.2967/jnumed.110.078741

\section{Errata}

In the article "Estimation of the ${ }^{18}$ F-FDG Input Function in Mice by Use of Dynamic Small-Animal PET and Minimal Blood Sample Data," by Ferl et al. ( J Nucl Med. 2007;2037-2045), one of the authors was inadvertently omitted from the by-line. The corrected by-line should read as follows: Gregory Z. Ferl, Xiaoli Zhang, Histo-Ming Wu, Michael C. Kreissl, and Sung-Cheng Huang. The authors regret the error.

In the article "18F-FDG PET After 2 Cycles of ABVD Predicts Event-Free Survival in Early and Advanced Hodgkin Lymphoma," by Cerci et al. ( $\mathrm{N} \mathrm{Nucl} \mathrm{Med}$. 2010;51:1337-1343), Table 5 contained a mistake. The 3-y event-free survival rate for PET-positive scans in the present study was $53.4 \%$ and not $24 \%$. The authors regret the error. 